\section{$\underset{\substack{\text { hommes } \\ \text { \& migrations }}}{ }$}

\section{Hommes \& migrations}

Revue française de référence sur les dynamiques

migratoires

$1313 \mid 2016$

1983, le tournant médiatique

\title{
Le meurtre du Bordeaux-Vintimille
}

\section{Céline Régnard}

\section{OpenEdition \\ Journals}

\section{Édition électronique}

URL : http://journals.openedition.org/hommesmigrations/3563

DOI : 10.4000/hommesmigrations.3563

ISSN : 2262-3353

\section{Éditeur}

Musée national de l'histoire de l'immigration

\section{Édition imprimée}

Date de publication : 1 janvier 2016

Pagination : 73-79

ISBN : 978-2-919040-34-6

ISSN : $1142-852 X$

\section{Référence électronique}

Céline Régnard, « Le meurtre du Bordeaux-Vintimille », Hommes \& migrations [En ligne], 1313 | 2016, mis en ligne le 01 janvier 2019, consulté le 16 mars 2020. URL : http://journals.openedition.org/ hommesmigrations/3563; DOI : https://doi.org/10.4000/hommesmigrations.3563 


\title{
UN FAIT DIVERS DANS LA «CHALEUR ET LE BRUIT 》 LE MEURTRE DE TOUFIK OUANNES À LA TÉLÉVISION (9 JUILLET 1983)
}

par CLAIRE SÉCAIL, chargée de recherche CNRS, Laboratoire Communication et politique, IRISSO (UMR 7170), CNRS/Université Paris Dauphine, PSL Research University.

\author{
Au mois de juillet 1983, un fait divers tragique endeuille \\ une cité de La Courneuve et fait la Une des médias. Si l'émotion \\ suscitée par le meurtre de Toufik Ouannes, 10 ans, est grande, \\ les explications du geste du tireur, un habitant de la cité \\ qu'indisposait le bruit ce soir-là, sont loin d'être unanimes. \\ Plusieurs récits cohabitent dans le traitement médiatique \\ de ce crime. Si son caractère raciste ne sera pas avéré, \\ il témoigne des tensions sociales récurrentes dans les quartiers \\ populaires face auxquelles se mobilise le pouvoir politique.
}

Ce samedi 9 juillet 1983, l'ambiance est à la fête dans la cité des 4000 à La Courneuve, à deux jours de la fin du ramadan. Vers $20 \mathrm{~h} 30$, un petit groupe d'enfants jouent au pied des barres d'immeubles. La journée a été chaude et les garçons s'amusent à allumer des pétards. Soudain, l'un d'eux titube, marche quelques mètres et s'écroule, touché par un projectile. L'enfant, Toufik Ouannes, âgé de 10 ans, meurt avant l'arrivée des secours. Les habitants de la cité, entre colère et émotion, réclament déjà de la police qu'elle déploie tous les moyens nécessaires pour retrouver le tireur meurtrier. La tension ne retombe que deux jours plus tard, le 12 juillet, quand intervient l'annonce de l'arrestation et des aveux du meurtrier. Ce père de famille de 50 ans, surveillant à la RATP, a une santé fragile. Ayant été victime d'un infarctus quelques semaines plus tôt, il explique avoir tiré avec la carabine à air comprimé de son fils pour mettre fin au chahut des enfants, excédé par le bruit des pétards qui l'empêchait de dormir. Il n'imaginait pas, raconte-t-il aux policiers, que son geste entraînerait la mort de l'enfant.

En pleine période estivale, à l'heure où les enfants rêvent de départ en vacances, le drame de La Courneuve fait aussitôt la Une des médias et, en premier lieu, des journaux télévisés. Comme une allumette qui craque, le fait divers réactive des inquiétudes latentes et des colères diffuses. Spectre du crime raciste intervenant dans le décor des grands ensembles, le meurtre de Toufik pouvaitil demeurer un fait divers isolé, sans ramifications 
politiques, sans conséquences sociales, sans effet de résonance médiatique? En ce début des années 1980, il est désormais difficile de distinguer un fait divers d'une question de société faisant l'objet d'un traitement fait-diversier ${ }^{1}$.

Pour comprendre ce que ce fait divers, replacé dans son contexte social, politique et journalistique, a cristallisé, il convient de dilater la temporalité pour analyser l'affaire Toufik à la lumière de trois généalogies de récits. La première est celle des récits de meurtres d'enfants, qui permet de comprendre la force des émotions collectives provoquées par le drame de la Courneuve. La deuxième se rapporte à celle des récits de "crimes racistes ", catégorie alors pénalement inexistante et journalistiquement flottante. Enfin, la dernière relève d'une catégorie de récits qui relève d'une montée en généralisation et d'une politisation des débats autour des émotions suscitées par les faits divers.

\section{Récits de «meurtres d'enfants »: une catégorie journalistique stabilisée}

En matière de récit criminel, lâge de la victime est souvent un élément central pour comprendre le déploiement des émotions collectives autour de certains faits divers ${ }^{2}$. Le meurtre d'enfants apparaissant comme la catégorie de crime la plus intolérable, il existe une sorte de loi d'airain qui veut que plus une victime est jeune, plus elle suscite de l'empathie auprès des publics et plus l'événement criminel tend à s'imposer à l'écran. Si l'on observe la morphologie des journaux télévisés de cette mijuillet 1983, l'affaire Toufik ne déroge pas à la règle et vient s'inscrire dans le sillage des affaires qui, depuis les années 1970 à la télévision, s'articulaient autour de la figure de l'enfant-victime. Après
Brigitte Dewèvre à Bruay-en-Artois (1972), MarieDolorès Rambla à Marseille (affaire Ranucci, 1974) ou Philippe Bertrand à Troyes (affaire Patrick Henry, 1976), ce 10 juillet 1983, c'est au tour de Toufik Ouannes, âgé de 10 ans, de faire la Une en incarnant l'innocence sacrifiée par la bêtise ou, comme le dira le présentateur Philippe Harrouard sur Antenne 2, la « lâcheté » des hommes ${ }^{3}$.

Dans les années 1970, la rubrique des faits divers détenait une légitimité encore fragile à la télévision. Les responsables de l'information avaient des réticences à accorder de l'importance dans les journaux télévisés à des récits qui semblaient valoriser les comportements criminels et risquaient d'influencer en particulier les jeunes publics. Reste que les meurtres d'enfants, en raison de l'indignation qu'ils soulèvent, comportent une forme de légitimité immédiate et intrinsèque au récit. Au fond, on ne craint plus de valoriser un criminel lorsque la compassion à l'égard de sa victime est suffisamment puissante pour nourrir une forme de rejet sans nuance du " monstre-meurtrier ». Dans les années 1970, les meurtres d'enfants ont permis la valorisation de la rubrique en jouant un rôle important pour bousculer les hiérarchies éditoriales figées des JT qui faisaient passer les informations politiques et internationales avant les faits divers ${ }^{4}$.

Les journaux télévisés à l'occasion de la mort de Toufik ne font pas exception : l'événement fait l'ouverture des éditions de mi-journée et de soirée pendant les deux jours qui suivent le drame, les 10 et 11 juillet. Le 12 juillet, au moment de l'arrestation et des aveux du meurtrier, l'affaire quitte cependant la Une, retrouve un rang secondaire, derrière une actualité marquée par une série d'attentats en Corse, un accident mortel d'ULM ou la tension militaire au Tchad. L'affaire Toufik s'inscrit bien dans cette généalogie de meurtres d'enfants

1. Alexandre Borrell, "Faits divers et faits de société dans la médiatisation des "banlieues" au 20 heures ", in Les Cahiers du journalisme, $n^{\circ} 17,2007, \mathrm{pp} .134-145$; "Les banlieues au “20 heures", de la promotion des grands ensembles aux Minguettes (1954-1981). Actualité, images et nation dans le journal télévisé ", thèse de doctorat d'histoire contemporaine de l'université d'Orléans, 2015 (dir. J. Garrigues) ; Claire Sécail, Le Crime à l'écran. Histoire du fait divers criminel à la télévision (1950-2010), Paris, Nouveau monde, 2010. 2. Anne-Claude Ambroise-Rendu, "La dangerosité du criminel sexuel sur enfant, une construction médiatique? ", in Le Temps des médias, $n^{\circ} 15,2010$, pp. $72-86$; Crimes et délits. Une histoire de la violence de la Belle Époque à nos jours, Paris, Nouveau monde éditions, 2006, p. 45 et sq. 3. INA, JT de 20 heures, A2, 10 juillet 1983. 4. Claire Sécail, op. cit. 
Portrait de Toufik Ouannes, brandi lors de la $3^{\text {e Marche }}$ pour l'égalité des droits en 1983 à la sortie d'une usine en région parisienne. ( ) PHOTO AGENCE IM'MÉDIA.

et l'âge du petit garçon est l'une des explications de la forte médiatisation du fait divers. Cependant, contrairement aux codes narratifs qui prévalent pour cette catégorie de récits, on observe des ruptures avec la mise en images classique et codifiée de ces événements. Alors même qu'il est la pierre angulaire du drame, Toufik semble finalement se dérober au récit qu'il génère. Quand, en 1976, le visage du jeune Philippe Bertrand emplissait l'écran et servait d'entrée émotionnelle directe dans le récit, le visage de Toufik apparaît finalement très peu à l'antenne, ne se glissant qu'à deux reprises et brièvement dans les reportages diffusés entre le 10 et le 12 juillet. Toufik n'y est d'ailleurs pas singularisé en tant que victime puisqu'il appa- raît en compagnie de son frère aîné Mohamed sur la photo de famille proposée aux journalistes Autre élément distinctif : l'enfant-victime n'a généralement pour identité que son seul prénom accompagné d'un vocable affectif destiné à renforcer son statut de victime : "le petit Philippe ", "la petite Marie-Dolorès ». Dans le cas de Toufik, ce vocable existe (« le petit Toufik », « le jeune Toufik », « un petit garçon de 10 ans sans histoire », etc.). Mais on repère d'autres formulations qui viennent particulariser l'enfant, le rapporter à un statut plus spécifique (" enfant de La Courneuve », " enfant de Nord-Africains ", " enfant d'origine algérienne ", et même " un jeune Algérien » ou un "petit Algérien de La Courneuve »), qui suggèrent que Toufik 
Ouannes n'est pas un enfant tout à fait comme les autres. Enfin, le temps des funérailles, habituellement un moment fort des récits de meurtres d'enfants puisqu'il permet, à travers le média amplificateur qu'est la télévision, de souder et d'élargir des communautés émotionnelles, est ici totalement absent de la médiatisation. Toufik n'aura pas de funérailles télévisuelles et le deuil de cet enfant restera privé, réduit aux proches et aux voisins.

\section{Récits de « crimes racistes »: une catégorie journalistique à géométrie variable}

Si l'on replace l'affaire Toufik dans une seconde généalogie de récits, on observe que les journaux télévisés se montrent particulièrement prudents, voire frileux, avec la notion de "crime raciste». Cette lecture journalistique trouve deux raisons principales. D’une part, l'incrimination de « crime raciste » n'est pas une catégorie définie dans le droit pénal. La « loi relative à la lutte contre le racisme », dite loi Pleven du $1^{\text {er }}$ juillet 1972, avait consolidé l'arsenal juridique existant dans ce domaine en précisant dans le droit de la presse les infractions telles que la provoca-

Toufik n’aura pas tion à la haine raciale, la difde funérailles télévisuelles famation et l'injure raciales et le deuil de cet enfant , ainsi que les actes de discrirestera privé, réduit minations. Hasard de l'acaux proches et aux voisins. tualité, les parlementaires français viendront compléter la loi Perven le 13 juillet 1983 - quatre jours après la mort de Toufik - en supprimant la possibilité d'exonérer l'auteur d'une discrimination raciale par un motif légitime. Mais, en matière de crime, il n'existe pas, en 1983, d'incrimination spécifique lorsqu'un acte commis comporte un mobile ou une motivation raciste. Le meurtre et l'assassinat étant déjà punis de la peine maximale, les députés français ont renoncé à venir introduire cette spécification dans le droit, arguant que cela n'avait aucune variation sur la sanction. Néanmoins, la multiplication de crimes racistes, notamment durant l'année 1983, ont poussé les parlementaires à franchir un pas supplémentaire en autorisant, par la loi du 3 janvier 1985, les associations reconnues dans le domaine de la lutte contre le racisme à se constituer partie civile dans les cas de crimes commis en raison de l'origine de la victime. Reste qu'en 1983, la légitimité de la parole associative n'est pas acquise. C'est ce que révèle la précaution de langage du présentateur Jean-Pierre Berthet lorsqu'il relaie le communiqué de l'Association de solidarité franco-arabe (ASFA, créée en 1967) tout en marquant sa distance à l'évocation d'un "drame raciste »: "Dans un communiqué, à la fin de cette matinée, l'Association de solidarité franco-arabe demande aux pouvoirs publics des mesures d'urgence afin d'enrayer, je cite, "l'engrenage de la violence qui peut conduire à de nouveaux drames racistes ${ }^{5}$ ". " En d'autres termes, l'information télévisée ne fait que refléter, ici, une lacune du droit français.

D'autre part, le " crime raciste ", parce qu'il n'a aucune base pénale, demeure une catégorie journalistique flottante, dépendante de la sensibilité du journaliste et des informations qu'il détient au moment où il relate les faits. Cette deuxième raison vient expliquer la prudence générale des journaux télévisés. Dans le cas Toufik, les faits restent mal connus pendant les deux jours de forte intensité du drame jusqu'à l'arrestation du meurtrier. Aucun détail sur l'identité du tireur n'ayant filtré en quarante-huit heures, les journalistes ignorent les raisons de son geste meurtrier et se contentent d'égrener une multifactorialité de causes, dont les deux dominantes accréditent l'hypothèse d'un coup de folie, la chaleur et le bruit. On voit alors se déployer un discours de rationalisation de la démesure meurtrière par la mise en évidence d'éléments thermo-acoustiques. Lorsqu'elle apparait, l'hypothèse du mobile raciste n'est jamais développée : "On ne sait toujours pas qui (...) quel homme rendu fou par le bruit, la fournaise, la haine des jeunes ou des Arabes (...) a tiré sur un enfant de 10 ans » (JT de 12 
heures, A2, 10 juillet). "Cette vague de chaleur n'est certainement pas étrangère au drame qui s'est produit hier soir dans une cité HLM de La Courneuve, en SeineSaint-Denis, dans la banlieue parisienne. La chaleur et le bruit » (JT de 13 heures, TF1, 10 juillet). "Chaleur, bruit, violence, fin du ramadan, racisme, entassement : tout a été dit depuis quarante-huit heures sur ce meurtre d'un petit garçon de 10 ans » (JT de 20 heures, A2, 11 juillet).

Le 12 juillet, le présentateur Hervé Chabalier invite un expert en "malfaisance des décibels " pour expliquer en quoi le bruit finit par « déboussoler des personnes déjà stressées par le rythme de vie actuelle». Il interroge son invité : «Alors quel est le phénomène qui fait qu'à un moment donné, parce que c'est trop fort et trop longtemps, on craque ${ }^{6}$ ? "Sur le plateau, Gabriel Moser, un des pionniers de la psychologie environnementale française, membre du Laboratoire de psychologie sociale appliquée de Paris- $V$, semble passer un pénible moment. Entre deux aveux d'incompétence pour répondre aux questions très pointues du présentateur à propos de l'affaire Toufik, il essaie d'expliquer que le bruit n'est qu'un facteur parmi d'autres et que, s'il cristallise les exaspérations, les conditions sociales et les difficultés économiques de vie sont des éléments bien plus déterminants.

Il est une exception notable à cette prudence générale des journalistes de télévision : Marcel Trillat. Quand ses confrères écartent le racisme ordinaire à coups de degrés Celsius et de décibels, lui se livre sur Antenne 2 à une cinglante dénonciation du crime raciste dès le 10 juillet, sans attendre les résultats de l'enquête. Que l'affaire Toufik repose ou non sur un mobile raciste, au fond peu importe aux yeux de ce journaliste militant qui a choisi d'endosser pour l'occasion un statut d'éditorialiste afin de présenter ce qui, selon lui, doit réellement donner matière à réflexion. Soucieux d'éveiller les consciences, d'alerter les publics, de freiner la montée des actes racistes et d'attirer l'attention sur les réalités sociales et économiques des quartiers populaires, Trillat balaie la multifactorialité des causes que présentent ses confrères pour n'en retenir qu'une, celle de l'intolérance qui met en danger les relations sociales. Pour cela, il « sérialise » l'affaire Toufik en la rapprochant de crimes récents qui évoquent pour lui le même mobile : «Un coup de feu qui claque. L'un de ces petits Gavroche qui se retrouve par terre, le nez dans le ruisseau. Il est 20 h 30 environ, à La Courneuve. Un peu plus tard, dans la nuit, à 4 h 50, près du foyer de jeunes travailleurs, à Saint-Ouen, cette fois, c'est la fête. Un ouvrier martiniquais de 32 ans sort dans la rue pour uriner. Il est peutêtre un peu éméché : il éclabousse la carrosserie d'une voiture. Le propriétaire du précieux véhicule sort un 6.35 et tire deux fois. Une balle dans le coeur. C'est fini. (...) Dans les deux cas, on ne sait pas trop qui a tiré. Dans les deux cas, cela pourrait être n'importe qui. Quelqu'un surgit quelques secondes de la nuit anonyme de l'intolérance ordinaire... racisme anti-jeune ou racisme anti-arabe, c'est la même chose... pour retourner aussitôt s'y dissimuler frileusement. (...) Alors on va maintenant sans doute trouver des explications. Vous savez bien : les banlieues surpeuplées, la crise, la canicule, le bruit, l'insécurité qui progresse et rend les gens nerveux, etc. Mais, au fait, à propos d'insécurité, un chiffre rarement cité par ceux qui brandissent cet épouvantail quand tout est bon pour quelques voix de plus aux élections : au moins 16 jeunes Maghrébins ont été assassinés en France depuis deux ans par des vigiles, des policiers ou des adeptes du 22 long rifle. Et puis il y a tous ceux qui ne passent pas à l'acte mais qui tuent à blanc avec les mots: "bougnoule", "négro", "pédé", "sale jeune"... Gavroche, ce matin, s'appelle Toufik? . " L'engagement de Trillat accentue la différenciation de traitement opérée par les deux chaînes de télévision : quasi clinique et réduite au factuel sur TF1 ; une mise en récit plus émotionnelle et porteuse d'une amorce de réflexion sur l'évolution de la société sur Antenne 2. 


\section{Généalogie des récits d'une « politisation des faits divers »}

La troisième généalogie de récits dans laquelle s'inscrit l'affaire Toufik s'apparente aux récits d'une "montée en généralisation »" et, par porosité, d'une politisation du débat autour des émotions faits-diversières. La politisation de l'affaire Toufik repose sur trois catégories d'enjeux.

Plus ténu dans le cas de l'affaire Toufik, le premier enjeu est celui du débat sur les armes à feu, traité à travers deux reportages entre le 10 et le 12 juillet. Si les journalistes ont en premier lieu parlé de la «poitrine perforée par une balle $e^{9}$ » du jeune Toufik, " mortellement blessé d'une balle de 22 long rifle $e^{10}$ ", il s'avère deux jours plus tard que le meurtrier René Aigueperse a utilisé une arme en vente libre, " une sorte de jouet pour grandes

Danslestitres desjournaux personnes mais qui peut télévisés, on ne parle d'ailleurs pas de «l'affaire Toufik» mais bien du « drame

de La Courneuve ». être très dangereuse à courte distance (...) : une carabine à air comprimé projetant des petits plombs de type Jumbo ${ }^{11}$ ", " une carabine de marque chinoise achetée à La Redoute ${ }^{12}$ ". Ainsi, la mort de Toufik se retrouve placée sous le sceau d'une tragique malchance et, pour cette raison, ne parvient pas à mettre en débat le thème de la vente d'armes à feu, alors que d'autres faits divers similaires posaient plus clairement la responsabilité des détenteurs d'armes de catégories dangereuses ${ }^{13}$.

Le second enjeu politique, qui n'est que partiellement posé par l'affaire Toufik, est celui de l'augmentation des violences motivées par l'intolérance et la haine à l'égard des immigrés. On l'a vu, le fait divers perd sa singularité et son autonomie pour se fondre dans le récit générique des actes de racisme à travers un procédé de sérialisation de deux drames (la mort de Toufik et celle du jeune adulte "martiniquais»). La juxtaposition de plusieurs faits autorise précisément les journalistes à développer une réflexion générale sur la société, à rendre dicibles des événements latents ou épars et à dégager l'intelligibilité des phénomènes de fond que révèle leur répétition, même si cela oblige un journaliste comme Marcel Trillat à forcer le trait pour faire entrer les faits dans des catégories prédéfinies.

Mais il y a des résistances à voir dans l'affaire Toufik un symbole de la lutte contre le racisme. Cette résistance provient des habitants de La Courneuve eux-mêmes qui, quelles que soient leurs origines, préfèrent afficher devant les caméras une solidarité collective, rejetant l'idée de racisme qui viendrait fragiliser davantage leur quotidien et contredire dans l'immédiat leur réclamation de justice plutôt que de vengeance. La seconde résistance provient des responsables politiques de gauche qui ne souhaitent pas donner du grain à moudre à la droite et à l'extrême droite en ravivant des arguments sécuritaires autour de la figure de l'immigré, quatre mois seulement après les élections municipales qui ont vu la première percée du FN et la conquête symbolique de la mairie de Dreux par une liste RPR-Front national (Jean-Pierre Stirbois devenant le premier maireadjoint frontiste).

La politisation la plus développée du fait divers se produit finalement sur le troisième thème : celui des grands ensembles. Dans les titres des journaux télévisés, on ne parle d'ailleurs pas de "l'affaire Toufik » mais bien du «drame de La Courneuve». La mort de Toufik n'a pas attendu son épilogue judiciaire pour devenir le procès des grands ensembles. Le fait divers cristallise les faillites des politiques publiques en matière de logement et se retrouve au cœur d'enjeux locaux et nationaux qui ne manquent pas de se chevaucher, comme en témoigne le débat télévisé organisé le 11 juillet par Claude Sérillon, qui voit s'opposer Georgina 
Dufoix, secrétaire d'État à la Famille, à la Population et aux Travailleurs immigrés, à Jean Tibéri, député RPR de Paris. Le présentateur d'Antenne 2 se justifie : "La mort violente d'un enfant de 10 ans, né à La Courneuve, ce n'est pas seulement une enquête policière. (...) C'est aussi une affaire de société, c'est-à-dire de choix politiques. " Alors que les enquêteurs recherchent un coupable, le journaliste s'intéresse aux " responsables » du fait divers : "Alors ce soir, vous, madame, parce que vous êtes en charge du sort des communautés immigrées, et puis vous, monsieur, puisque vous présidez l'Office des HLM concerné, est-ce que vous ne vous sentez pas un petit peu responsables, l'un et l'autre, de ce qui s'est passé $e^{14}$ ?"

Différents reportages ont certes donné la parole aux architectes qui n'ont pas manqué de faire le procès urbanistique des grands ensembles ${ }^{15}$. Mais ces professions techniques ne suffisent pas à endosser les échecs des politiques du logement successives $^{16}$. C'est donc vers les autorités politiques que les journalistes se tournent. Sur le plateau, la confrontation partisane se double d'enjeux locaux opposant la Ville de Paris à la mairie communiste de La Courneuve, puisque Jean Tibéri est également président de l'Office HLM de la Ville de Paris qui est alors propriétaire de la cité des 4000 de La Courneuve. Le contexte immédiat ne joue pas en faveur de la secrétaire d'État : même si l'enquête vient d'aboutir sur l'identification du meurtrier de Toufik, la journée a été jalonnée de violences qui ont éclaté dans la cité. Jean Tiberi en profite pour imposer sa lecture sécuritaire, utilisant le drame de La Courneuve pour défendre la nécessité de renforcer la sécurité des personnes, d'augmenter la présence policière sur le terrain et finalement pour dénoncer «la politique incohérente de l'immigration":
"À un moment on laisse entrer tout le monde, puis le lendemain on prend des mesures répressives(...). Tout ça est très grave et n'est pas très sérieux ${ }^{17}$. " Face à lui, Georgina Dufoix cherche d'abord à rappeler

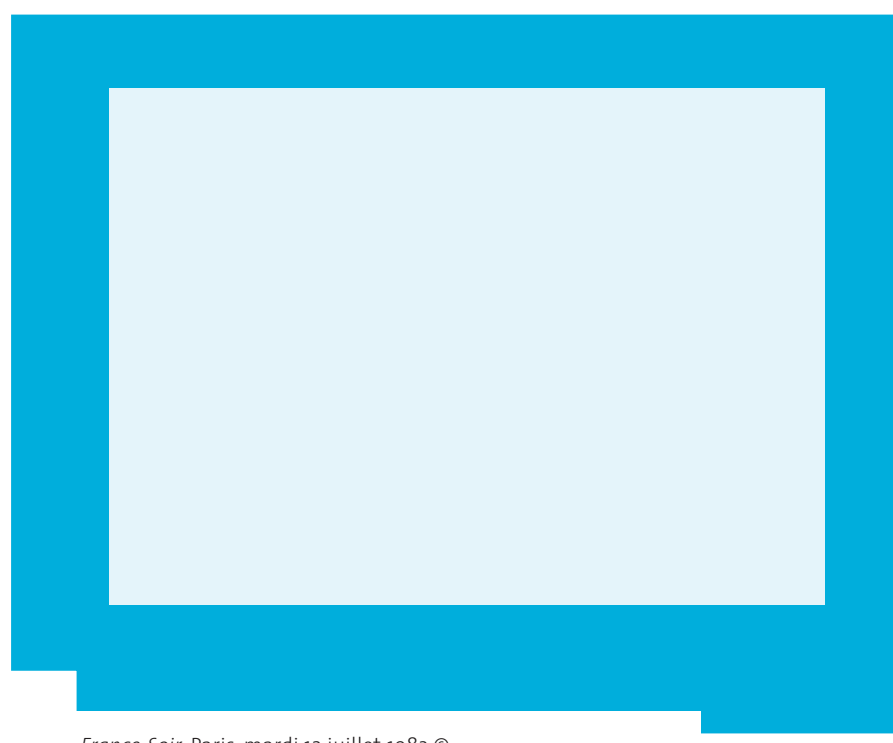

France-Soir, Paris, mardi 12 juillet 1983 @

les réalités économiques et sociales qui fragilisent les grandes cités. Mais elle a bien du mal à ne pas entrer dans la rhétorique sécuritaire de son adversaire et finit par renvoyer l'image d'une politique gouvernementale équilibriste de l'immigration : "Les hommes et les femmes qui sont sur notre territoire, pour la majorité d'entre eux, resteront sur notre territoire. Ils ont donc besoin d'une politique particulière à leur égard, d'insertion vers l'habitat, vers l'éducation, etc. Mais nous ne pouvons pas accepter que des clandestins rentrent aujourd'hui sur notre territoire. Et, par conséquent, nous aurons une très grande fermeté à l'égard de celles et de ceux qui viennent dans notre pays pour trouver du travail, car il n'y en a pas aujourd'hui ${ }^{18}$." 


\section{La réponse du pouvoir politique}

Pour désamorcer le climat sécuritaire et raciste qui s'engage et retrouver la maîtrise de l'agenda, le chef de l'État choisit d'entrer lui-même en scène. Quinze jours après le drame, François Mitterrand effectue une visite surprise de trois heures à La Courneuve, à l'abri des caméras et dans le cadre d'un déplacement en banlieue parisienne organisé par les animateurs de l'association Banlieue $89^{19}$. Les journalistes n'ayant pas été avertis, il n'existe pas d'images de la visite dans les appartements délabrés de La Courneuve ${ }^{20}$. En revanche, les caméras ont recueilli le Dans les années 1980, témoignage des habitants: sous la pression croissante des médias et de l'opinion publique, l'autorité politique se voit désormais acculée à réagir publiquement et rapidement aux événements dramatiques de l'actualité pour montrer sa capacité à comprendre les problèmes

d'y apporter une réponse soulevés et son souci institutionnelle. "On était au café et on a vu du monde. D'un coup on s'est dit il doit y avoir comme d'habitude une bagarre ou quoi que ce soit. Et on voit arriver le président, d'un coup! C'est vraiment une surprise quion a eue, que le président était là. Surtout à La Courneuve, dans la situation où on se trouve à l'heure actuelle»; " Je lui ai offert un verre, il était très content. On a pris

des photos aussi ensembles Je suis très contente ». La télévision capte aussi les propos du président de la République quelques heures plus tard à la Bourse du Travail, en Seine-Saint-Denis, qui donne alors son sentiment sur l'état de délabrement des cités, soulignant "l'accueil chaleureux et les relations humaines qui, d'une façon générale, sont bonnes". Surtout, il vient faire la promotion de son futur plan de réhabilitation des quartiers, au cœur des missions de l'association Banlieue 89 : «Donc maintenant, ce qui dépend pour une part du gouvernement - mais pas seulement du gouvernement -, il faut réhabiliter. Dans ce terme un peu compliqué qui n'est pas toujours bien perçu par l'habitant: rendre ce quartier agréable... commençons par dire "habitable" pour que... on y vive mieux... Que l'on ait envie de... comment dirais-je... d'y élever sa famille, de ne pas avoir ses enfants pratiquement abandonnés, sans véritables espaces verts, sur des terrains comme on dit "vagues"... qui sont bien vagues. Ceci est un effort de reprise en main que j'ai décidé d'entreprendre. "À la suite de cette visite, François Mitterrand créera une mission interministérielle intitulée "Banlieue 89 »: l'objectif est de lancer des consultations pour initier des projets en direction des architectes, urbanistes, paysagistes et plasticiens de rénovation afin de réhabiliter l'habitat des grands ensembles à l'horizon de l'année $1989^{21}$.

L'explication de la mort de Toufik par un excédent de degrés Celsius prend une autre dimension à travers la mise en place immédiate d'un nouveau plan " anti-été chaud " dans les jours qui suivent. Ce plan prévoit l'adoption de 23 conventions signées avec des associations de jeunesse et d'éducation populaire, en particulier dans les quartiers sensibles des grandes métropoles (Lyon, Paris, Marseille). La Courneuve fait partie du dispositif. De ce point de vue, l'affaire Toufik n'est pas seulement héritière des périodes antérieures (années 19601970), quand les responsables politiques cherchaient encore à isoler leurs actions politiques des émotions collectives liées à des faits divers. Dans les années 1980, sous la pression croissante des médias et de l'opinion publique, l'autorité politique se voit désormais acculée à réagir publiquement et rapidement aux événements dramatiques de l'actualité pour montrer sa capacité à comprendre les problèmes soulevés et son souci d'y apporter une réponse institutionnelle. 


\section{Conclusion}

L'affaire Toufik est-elle le récit de la mort d'un enfant de 10 ans, le récit de la mort d'un enfant d'origine algérienne ou le récit de la mort d'un Gavroche d'une cité de banlieue parisienne? Sans doute les trois à la fois, comme le montre l'analyse des journaux télévisés. Pourtant, l'affaire ressemble à un fait divers qui aurait subi des contorsions non négligeables pour entrer dans des catégories incertaines (en particulier celle du crime raciste) ou des récits codifiés (ceux des meurtres d'enfants).

Lépilogue judiciaire invalide définitivement l'hypothèse du crime raciste. Lorsque René Aigueperse se présente le 21 avril 1986 devant la cour d'assises de Bobigny, il est, selon l'arrêt de renvoi, " jugé pour un acte d'homicide dépourvu de tout caractère raciste ». Pour le meurtre, il est néanmoins condamné à cinq ans d'emprisonnement, dont deux avec sursis. Un détail révèle, par ailleurs, l'ambivalence médiatique : en rappelant les circonstances du drame, les chroniqueurs judiciaires expliqueront que les enfants jouaient avec les pétards en raison de l'approche des fêtes du 14 juillet sans mentionner qu'il s'agissait de la fin du Ramadan. À l'annonce du verdict, la mère de Toufik sera également présente sur les marches du palais, soucieuse d'empêcher la foule présente de faire éclater une colère pour dénoncer l'éventuelle tolérance de la justice à l'égard des comportements racistes. Comme en 1983, elle rejette avec fermeté les tentatives de faire de son enfant un martyr de la haine raciale. Isolée, l'affaire Toufik ne saurait à elle seule constituer un tournant médiatique de la question de l'immigration en France. Cette question y est même relativement désamorcée au fil des reportages, placée sous la tutelle d'un débat sur la politique du logement et, dans une moindre mesure, celle de l'immigration. Mais resituée à l'échelle de l'année 1983, réintégrée dans une série de faits divers tragiques, elle est cependant parvenue à faire bouger des lignes non seulement émotionnelles mais également politiques ${ }^{22}$. Le 26 décembre, lorsque le présentateur Bernard Rapp propose, à l'heure des regards rétrospectifs sur l'année 1983, d'illustrer ce qu'il identifie comme les "grandes tendances » de l'année écoulée, il retient, loin des humeurs de réjouissances, ce "phénomène des plus troublants $^{23}$ » qu'est le racisme. C'est à Marcel Trillat qu'a été confié le reportage. Le journaliste a choisi d'illustrer ce thème à travers l'évocation de deux faits divers ayant marqué l'année : l'affaire Toufik et le crime du Bordeaux-Vintimille, drame dont le mobile raciste ne fait, lui, aucun doute ${ }^{24}$. Choisissant de tourner son reportage dans le décor d'un train en marche, il livre son analyse, qui n'a pas varié depuis juillet : "Touché au coeur, Toufik vient mourir au pied de l'immeuble. Sur ce banc de pierre. À qui la faute? La canicule, peut-être ? l'entassement dans ces termitières de banlieue? le chômage? l'insécurité ?.... Et puis la haine, le refus de côtoyer celui qui diffère. Et l'on tue pour cela de plus en plus. Trente fois en $1983^{25}$. L La statistique vient ici remplir la fonction de contribuer à construire un enjeu de société que le fait divers n'avait fait qu'amorcer tout en lui donnant un point d'appui. Et de rappeler le jeune Toufik à la mémoire de chacun pour qu'un reportage puisse servir de tombe à toutes les victimes de la haine et des préjugés. 